\title{
Predictive value of red blood cell distribution width for mortality in patients with acute pancreatitis: A systematic review and meta-analysis
}

\author{
Azita Ganji ${ }^{1,2}$, Abbas Esmaeilzadeh ${ }^{1,2}$, Omid Ghanaei ${ }^{1,2}$, Amin Saberi $^{3 *}$, Danial Taherzadeh ${ }^{4}$, Soodabe Sazgarnia ${ }^{5}$, \\ Zohre Mayabi Joghal ${ }^{5}$, Mahboube Zirak ${ }^{6}$, Salman AbdolahRamazani ${ }^{7}$, Leili Zarifmahmoudi ${ }^{8}$
}

Received: 22 Dec 2016

Published: 23 Dec 2017

\section{Abstract}

Background: Red blood cell distribution width (RDW) is a quantitative measure of variability in the size of circulating erythrocytes. It has been recently identified as a prognostic marker in several diseases including acute pancreatitis (AP). In this systematic review the prognostic value of RDW in predicting mortality of AP patients will be assessed.

Methods: PubMed, Scopus, EMBASE, and ISI databases were searched until September 2016 using the following search strategy: (pancreatitis OR pancreatitides) AND (RDW OR "red cell distribution width" OR "red blood cell distribution width" OR anisocytosis). Four authors independently reviewed the retrieved articles. Studies were included if they had evaluated the association between RDW value and mortality of acute pancreatitis patients. Case reports, comments, letters to the editor, reviews, study protocols, and experimental studies were not included. Data abstraction and quality assessment for the included studies was independently performed by two authors. Quality of studies was assessed using Oxford Center for Evidence-Based Medicine checklist for prognostic studies. Data were synthesized qualitatively, and a meta-analysis was performed on the diagnostic performance of RDW to predict mortality in AP patients.

Results: Seven studies (976 patients) were included in the systematic review. Six studies reported a statistically significant association between RDW value and mortality. Meta-analysis was performed on four studies (487 patients) using a bivariate model and a summary receiver operating characteristic (sROC) curve was plotted with an area under the curve (AUC) of 0.757 . The pooled diagnostic odds ratio (DOR), sensitivity and specificity was 19.51 (95\% CI: 5.26-72.30), 67\% (95\% CI: 51\%-80\%) and 90\% (95\% CI: 73\%-96\%), respectively.

Conclusion: RDW is an easy to use and an inexpensive marker with a moderate prognostic value to predict death in AP patients. Clinicians should be more alert when a patient with AP has an increased RDW. Investigation of possible combinations of other prognostic markers with RDW is recommended.

Keywords: Red blood cell distribution width, Acute pancreatitis, Prognosis, Mortality, Systematic review, Meta-analysis Copyright@ Iran University of Medical Sciences

Cite this article as: Ganji A, Esmaeilzadeh A, Ghanaei O, Saberi A, Taherzadeh D, Sazgarnia S, Mayabi Joghal Z, Zirak M, AbdolahRamazani S, Zarifmahmoudi L. Predictive value of red blood cell distribution width for mortality in patients with acute pancreatitis: A systematic review and metaanalysis. Med J Islam Repub Iran. 2017 (23 Dec);31:124. https://doi.org/10.14196/mjiri.31.124

\section{Introduction}

Acute pancreatitis (AP) is an acute inflammatory disease induced by activation of pancreatic enzymes and is

Corresponding author: Amin Saberi, amnsbr@gmail.com

1. Gastroenterology and Hepatology Research Center, Mashhad University of Medical Sciences, Mashhad, Iran.

2. Department of Gastroenterology and Hepatology, Faculty of Medicine, Mashhad University of Medical Sciences, Mashhad, Iran.

3. Student Research Committee, Faculty of Medicine, Mashhad University of Medical Sciences, Mashhad, Iran.

4. Biochemistry \& Nutrition Research Center, Department of Biochemistry, Faculty of Medicine, Mashhad University of Medical Sciences, Mashhad, Iran.

5. Student Research Committee, Faculty of Medicine, Mashhad Azad University of Medical Sciences, Mashhad, Iran.

6. Student Research Committee, Faculty of Medicine, Sabzevar University of Medica Sciences, Sabzevar, Iran.

7. Department of Medicinal Chemistry, School of Pharmacy, Mashhad University of Medical Sciences, Mashhad, Iran.

8. Nuclear Medicine Research Center, Mashhad University of Medical Sciences, Mashhad, Iran. caused by a variety of etiologies, most notably gallstones and alcohol [4]. The incidence of AP is $150-420$ and 330-

$\uparrow$ What is "already known” in this topic:

RDW has been shown to be a prognostic marker in a variety of diseases. Several original studies have evaluated its prognostic role in acute pancreatitis and reported diverse results.

$\rightarrow$ What this article adds:

Most of the included studies identified RDW as a significant predictor of mortality in acute pancreatitis. Our metaanalysis shows a moderate prognostic performance of RDW in predicting mortality in acute pancreatitis. 
430 cases per million in the United Kingdom and the United States, respectively [5]. AP can be categorized into mild AP (MAP) to severe AP (SAP) and is a cause of acute abdomen. AP can be complicated with systemic inflammatory response syndrome and multiple organ failure. The mortality rate is in a range of $3.8 \%$ to $7 \%$ in AP patients and about $40-70 \%$ in severe cases [8]. Therefore, the diagnosis of AP and prediction of its severity is important for early management of the patients in order to decrease the rates of morbidity and mortality.

Several parameters and scoring systems, such as Ranson criteria and the Acute Physiology and Chronic Health Evaluation II (APACHE II) score have been used to predict the severity of AP and its mortality [9, 10]. Ranson criteria is required to be assessed 48 hours after the onset of AP and may not be useful for early prediction of AP mortality [7]. Ranson and APACHE II scores are complex, unreliable and difficult to use in clinical practice [11]. Therefore, it is important to identify a simple, easy to use, sensitive, and early marker to predict the mortality associated with AP.

Red blood cell distribution width (RDW), reported as a part of the complete blood count test, is a quantitative measure of variability in the size of circulating erythrocytes [13]. RDW is mainly used as a diagnostic method to differentiate thalassemia from iron deficiency anemia [14]. However recent studies have reported RDW as a predictor of mortality in cardiac diseases, stroke, pulmonary hypertension, peripheral artery disease, inflammatory and infectious conditions, liver disease, celiac disease, colon cancer, coronary artery disease and pulmonary embolism [15-23].

\section{Objectives}

RDW has recently been identified as a prognostic marker for AP in several studies. Therefore we performed a systematic review and meta-analysis to evaluate the association of RDW value with mortality and to investigate its prognostic performance as a predictor of mortality in AP patients.

\section{Methods \\ Search strategy for identification of studies}

This systematic review and meta-analysis is performed according to the PRISMA (Preferred Reporting Items for Systematic Reviews and Meta-Analyses) guideline [24].

PubMed, Scopus, EMBASE, and ISI databases were searched comprehensively for relevant studies from inception to September 2016, using the following search strategy: (pancreatitis OR pancreatitides) AND (RDW OR "red cell distribution width" OR "red blood cell distribution width" OR anisocytosis). No language or time limitation was applied. The references lists of the included studies were also hand-searched to identify further relevant citations and prevent missing articles.

\section{Inclusion and exclusion criteria}

Studies were included if they had evaluated the association between RDW value and mortality of AP patients by either comparing RDW values between survivors and non- survivors or assessing the diagnostic performance of RDW to predict mortality. Mortality was not limited to any time period. Case reports, comments, letters to editor, reviews, study protocols, and experimental studies were not included. Meeting abstracts were not excluded if reported sufficient data. When duplicate articles were suspected, the one containing more relevant data was included. Studies were included in the meta-analysis if they had reported diagnostic $2 \times 2$ tables or sensitivity and specificity of RDW value to predict mortality in AP patients.

\section{Methods of the review}

\section{Selection of studies}

Four authors (MZ, SA, SS, ZM) independently reviewed the retrieved articles to identify relevant studies. Discrepancies were resolved by consensus.

\section{Data extraction and Quality assessment}

Relevant data including patients characteristics, survival rates, RDW measurement methods and statistical analyses results were independently extracted by two authors using a data extraction form. Methodological quality of the included studies was assessed independently by two authors using Oxford Center for Evidence-Based Medicine checklist for prognostic studies [25].

\section{Statistical analysis}

Studies were included in the meta-analysis if they had reported the diagnostic performance of RDW to predict mortality. The $2 \times 2$ tables, including number of true positives (TP), false negatives (FN), true negatives (TN), and false positives (FP) was designed for each study based on their reported sensitivity, specificity, and mortality rate.

When there is no common cut-off between studies, as is the case in this meta-analysis, the test accuracy should be summarized as a sROC curve and its corresponding AUC and not by separate pooling of sensitivity and specificity values [26]. Therefore, the meta-analysis was performed using a bivariate model that has the advantage of analyzing sensitivity and specificity jointly [27]. R programming language ( $\mathrm{R}$ Foundation, Vienna, Austria) with mada package was used to perform this meta-analysis [28]. The sROC curve and its AUC, pooled sensitivity and specificity as well as the $95 \%$ confidence region (depicting the precision of pooled estimate) and $95 \%$ prediction region (illustrating the likely range that true values of a future study would lie) were reported [29]. Additionally, a separate meta-analysis was performed on the DOR using the same software package and was reported as a forest plot.

Heterogeneity was evaluated by visual inspection of ROC space and was considered high when the $95 \%$ prediction region was much larger than $95 \%$ confidence region [29]. $\mathrm{I}^{2}$ index and Cochrane $\mathrm{Q}$ test are not useful for identifying heterogeneity in diagnostic test accuracy metaanalyses and were not used in this study [29]. Heterogeneity can partly be explained by the different cut-off values (i.e. threshold effect). Meta-DiSC 1.4 was used to evaluate this effect using a Spearman's correlation between logit of true positive and false positive rates. Threshold effect exists when this correlation is statistically significant [30]. 
We also planned to perform subgroup analyses when there were at least two studies in each subgroup. The level of significance was set at $\mathrm{p}<0.05$.

\section{Results}

\section{Study selection}

A summary of study selection process (i.e. PRISMA flowchart) is shown in Fig. 1. The initial search identified 25 non-duplicate records. After screening the titles and abstracts, 14 studies were excluded. For the remaining 11 studies, full-texts were retrieved to assess their eligibility to be included in the systematic review. Four studies were excluded because two were duplicates and two were not relevant. Seven studies were finally included in our systematic review $[1-3,6-8,12]$. Three studies were not in English [2, 3, 12]. No additional eligible studies were found after reviewing the references lists of the included studies.

\section{Characteristics of the included studies}

Characteristics of the included studies and their results are summarized in Table 1. A total of 976 patients (525 female and 448 male) from four different countries (Turkey, China, Poland and Czech Republic) were included in this systematic review. Average age of the patients was in a range of 46.5 to 56.5 years. Biliary occlusion was the most common cause of AP (ranging from $35.8 \%$ to $73.5 \%$ of cases) in the five studies that had reported the etiologies. With the exception of one study [12], all studies included both mild and severe forms of AP, although in one study [1] the severity of the disease was not reported. Five studies were designed as retrospective cohorts $[1,3,7,8$, 12] and two as prospective cohorts [2,6]. RDW value was determined using Sysmex hematology analyzer (Sysmex Corp., Kobe, Japan) in three studies [2, 6, 8], and in four studies the measurement devices were not mentioned [1, $3,7,12]$. RDW was measured in the first 24 hours after

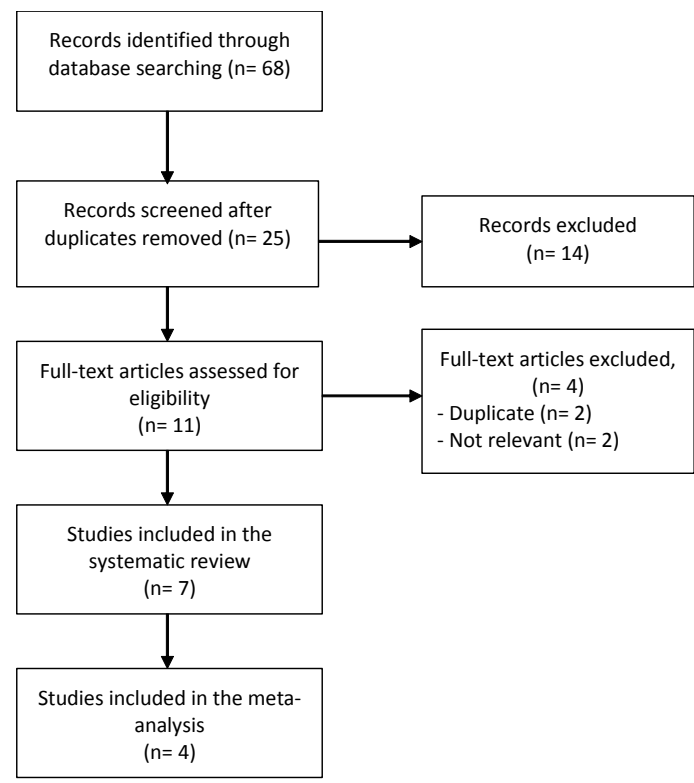

Fig. 1. Flow chart summarizing the process of study selection

admission in four studies [3, 6-8] but one study measured it on days 3, 5 and 7 [2], and the time of RDW measurement was not reported in two studies [1, 12].

Duration of follow-up in the included studies varied from 24 hours to 3 months and was not reported in two studies [3, 12]. Survival rates were in a range of $64.04 \%$ to $87.25 \%$. The study of Yang et al. [12], which included only severe AP patients, was the one with the highest mortality rate. The causes of mortality were limited to pancreatitis and its complications in four studies $[2,3,7,8]$ and were not reported in three studies $[1,6,12]$.

\section{Methodological quality of included studies}

Quality of the included studies was assessed using Oxford Center for Evidence-Based Medicine checklist for
Table 1. Summary of findings

\begin{tabular}{|c|c|c|c|c|c|c|c|}
\hline \multirow[t]{2}{*}{ First Author, Year, Country } & \multirow[t]{2}{*}{$\mathrm{n}$} & \multirow{2}{*}{$\begin{array}{c}\text { Age } \\
\text { (years) } \\
\operatorname{sex}(\mathrm{F} / \mathrm{M})\end{array}$} & \multirow[t]{2}{*}{$\begin{array}{l}\text { Survival/ } \\
\text { death }\end{array}$} & \multicolumn{3}{|c|}{$\begin{array}{c}\text { Difference in \%RDW between survivors and } \\
\text { non-survivors }\end{array}$} & \multirow[t]{2}{*}{$\begin{array}{l}\text { Logistic } \\
\text { regression }\end{array}$} \\
\hline & & & & \%RDW in survivors & $\begin{array}{c}\text { \%RDW in } \\
\text { non-survivors }\end{array}$ & $\mathrm{p}$ & \\
\hline Senol, 2013, Turkey ${ }^{[1]}$ & 102 & $\begin{array}{c}56.5 \\
59 / 43\end{array}$ & $89 / 13$ & $13.3(12.5-14.3)^{\dagger}$ & $15.6(14-21)^{\dagger}$ & $\begin{array}{l}<0.001^{*} \\
0.001^{*}, 1\end{array}$ & - \\
\hline Kolber, 2013, Poland ${ }^{[2]}$ & 40 & $\begin{array}{c}46.6 \\
16 / 24\end{array}$ & $34 / 6$ & $13.6^{\dagger}$ & $14.3^{\dagger}$ & $<0.05^{*}$ & - \\
\hline Floreánová, 2014, Czech Republic ${ }^{[3]}$ & 159 & $\begin{array}{c}56 \\
68 / 91\end{array}$ & $140 / 19$ & - & - & - & - \\
\hline Yao, 2014, China ${ }^{[6]}$ & 106 & $\begin{array}{c}51.2 \\
54 / 52\end{array}$ & $98 / 8$ & $12.98 \pm 1.04^{\wedge}$ & $14.2 \pm 0.72^{\wedge}$ & $0.002^{\$}$ & - \\
\hline Gülen, 2015, Turkey ${ }^{[7]}$ & 332 & $\begin{array}{c}53.1 \\
212 / 120\end{array}$ & $318 / 14$ & $13.7(12.9-14.5)^{\dagger}$ & $14.7(13.7-15.6)^{\dagger}$ & $0.201^{*}$ & not significant ${ }^{2}$ \\
\hline Wang, 2015, China ${ }^{[8]}$ & 120 & $\begin{array}{c}51.2 \\
70 / 50\end{array}$ & $104 / 16$ & $12.82 \pm 0.95^{\wedge}$ & $14.31 \pm 0.85^{\wedge}$ & $<0.001^{*}$ & - \\
\hline Yang, 2016, China ${ }^{[12]}$ & 117 & 54.2 & $46 / 68$ & $13.4+-1.5^{\wedge}$ & $16.6+-1.7^{\wedge}$ & $0.001^{\$}$ & $\begin{array}{l}\text { OR } 1.170(95 \% \\
\text { CI: } 1.004-1.325, \\
\quad P=0.008)^{3}\end{array}$ \\
\hline $\begin{array}{l}\text { A mean } \pm \text { SD } \\
\dagger \text { median (range) } \\
\$ \text { Student's t-test } \\
\text { * Mann-Whitney U test } \\
{ }^{1} \text { Multivariate Mann-Whitney with age, BU } \\
{ }^{2} \text { Multivariate logistic regression with Balt }\end{array}$ & $\begin{array}{l}\text { elet c } \\
\text { assifi }\end{array}$ & $\begin{array}{l}\text { WBC cou } \\
\text { n, HAPS s }\end{array}$ & $\begin{array}{l}\text { umin as } \\
\text { LR, age }\end{array}$ & $\begin{array}{l}\text { ding variables } \\
\text { s mellitus and systolic }\end{array}$ & rtension as & ariables & \\
\hline
\end{tabular}




\begin{tabular}{|c|c|c|c|c|}
\hline First Author, Year & $\begin{array}{l}\text { inclusion at a } \\
\text { common point }\end{array}$ & Duration of follow-up or hospital stay & $\begin{array}{l}\text { Method of outcome evaluation } \\
\text { / Blind outcome evaluation }\end{array}$ & $\begin{array}{l}\text { Adjustment for con- } \\
\text { founding variables }\end{array}$ \\
\hline Senol, $2013^{[1]}$ & Yes & Median of 6 (1-88) days & Death of all causes / NA & Yes \\
\hline Kolber, $2013^{[2]}$ & Yes & $\begin{array}{l}\text { Median of } 6.5(5-10) \text { days in mild and } 20.5 \\
(12-176) \text { in severe or complicated cases }\end{array}$ & Death of all causes / NA & No \\
\hline Floreánová, $2014^{[3]}$ & Yes & Not reported & Death of all causes / NA & No \\
\hline Yao, $2014^{[6]}$ & Yes & Up to 3 months & Death of all causes / NA & No \\
\hline Gülen, $2015^{\text {[7] }}$ & Yes & First 24 hours after admission & Death of all causes / NA & Yes \\
\hline Wang, $2015^{[8]}$ & Yes & Up to 3 months & Death of all causes / NA & No \\
\hline Yang, $2016^{[12]}$ & Yes & Not reported & Death of all causes / NA & Yes \\
\hline
\end{tabular}

Table 3. Diagnostic data of the studies included in the meta-analysis. TP: true positives, FP: false positives, FN: false negatives, TN: true negatives First author, Year

Senol, $2013^{[1]}$

Floreanova, $2014^{[3]}$

Yao, 2014 [6]

Wang, $2015^{[8]}$

\begin{tabular}{cc}
\hline Total \\
102 \\
159 \\
106 \\
120
\end{tabular}
Number of patients

prognostic studies and is reported in Table 2. Confounding variables were adjusted in three studies $[1,7,12]$, one of which [7] reported no association between RDW value and mortality.

\section{RDW and mortality}

Using various statistical analyses, in six studies a significant association between RDW value and mortality was observed [1-3, 6, 8, 12]. The only study which reported no such association was the one with the highest number of participants [7]. Differences in mean or median of RDW values between survivors and non-survivors were evaluated using Mann-Whitney U test or Student's t-test in six studies $[1,2,6-8,12]$ which were statistically significant in five $[1,2,6,8,12]$. Mean or median of RDW values in survivors ranged from $12.8 \%$ to $13.7 \%$ compared to a range of $14.2 \%$ to $16.6 \%$ in non-survivors.

Two studies performed multivariate logistic regression to assess the prognostic performance of RDW value as a predictor of mortality. One of them [12] reported a statistically significant odds ratio of 1.170 (95\% CI: $1.004-$ 1.325) for RDW, but in the other one [7] the logistic regression was not significant, and the odds ratio was not reported. Four articles (487 patients) reported the diagnostic accuracy of RDW to predict mortality and were included in the meta-analysis [1, 3, 6, 8]. Cut-off values for RDW varied between studies in a range of $13.2 \%$ to $14.8 \%$ (Table 3 ).

\section{Results of the statistical analysis}

The area under the sROC curve generated by the bivariate model was 0.757 , indicating a moderate prognostic accuracy of RDW to predict mortality (Fig. 2). The pooled diagnostic odds ratio was 19.51 (95\% CI: 5.26-72.30) (Fig. 3). The pooled sensitivity and specificity was $67 \%$ (95\% CI: 51\%-80\%) and 90\% (95\% CI: 73\%-96\%) respectively (Fig. 2).

The large area of $95 \%$ prediction region in the sROC area indicates a high heterogeneity between studies (Fig. 2).
There was no significant correlation between logit of true positive and false positive rates $(r=-0.209, \mathrm{p}=0.729)$ indicating that the heterogeneity cannot be explained by the threshold effect. Due to the small number of the included studies subgroup analysis was not performed.

\section{Discussion}

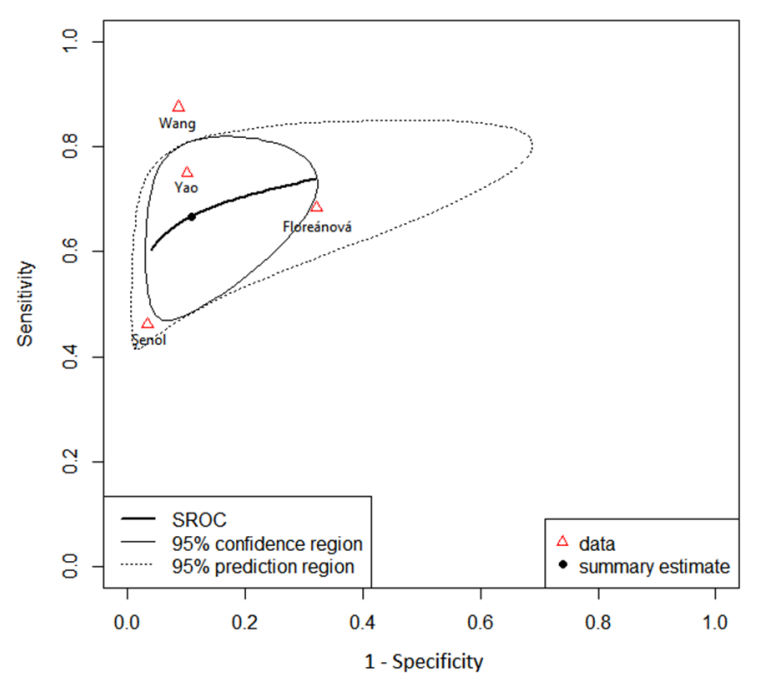

Fig. 2. Summary receiver operating characteristic curve of the included studies

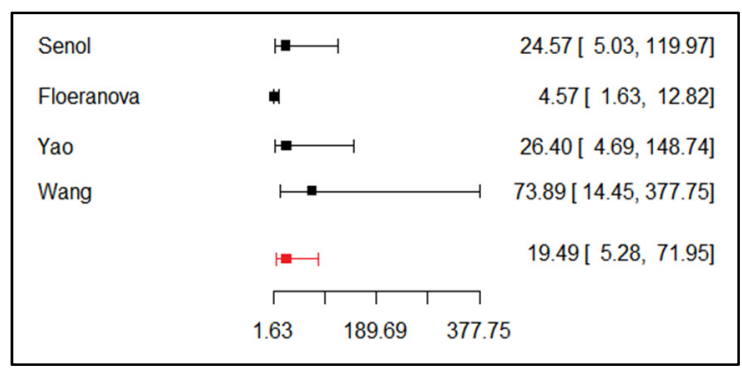

Fig. 3. Forrest plot of diagnostic odds ratio pooling 
In this systematic review, six out of seven studies reported a statistically significant association between RDW and mortality in AP patients. RDW values were greater in the non-survivors than in survivors. The sROC curve and a pooled diagnostic odds ratio of 19.51 (95\% CI: 5.2672.30) indicate a moderate prognostic performance of RDW to predict mortality in AP patients. The pooled sensitivity of $67 \%(95 \% \mathrm{CI}: 51 \%-80 \%)$ and specificity of 90\% (95\% CI: 73\%-96\%) show that in predicting mortality RDW is more specific rather than sensitive; and specificity is more important for a prognostic marker.

A wide variety of clinical, biochemical and imaging parameters as well as scoring systems such as Ranson, APACHE II, and BISAP has been utilized to predict the prognosis of AP patients [1]. However, most of these parameters are complex, unreliable and difficult to use in clinical practice [7]. Therefore it is important to identify a simple, inexpensive, noninvasive and sensitive marker to predict the mortality of AP patients.

RDW has several advantages as a prognostic marker. It is a component of the complete blood count test which is fairly inexpensive and is routinely tested in patients with suspected AP [1, 31]. Additionally, it is accessible in many hospitals and a very short time is required for the results to be reported [32]. Moreover, in most of the included studies RDW was measured in the first day of admission, and therefore it can be an early prognostic factor compared to Ranson score which is required to be assessed $48 \mathrm{~h}$ after the onset of AP.

The underlying mechanism for the observed association between RDW and mortality in AP is not clear. One suggested explanation is that RDW value is a reflection of the inflammatory status which is associated with higher mortality [33]. Inflammation increases RDW by promoting death and inhibiting maturation of RBCs, allowing reticulocytes to enter the circulation [34]. Additionally, inflammation changes the morphology of RBCs by altering their membrane glycoproteins and ion channels [34].

\section{Limitations}

Most of the included studies were inherently limited by their retrospective nature. Additionally, confounding variables were accounted for in only three studies, reflecting a relatively low quality of the included studies.

We aimed to reduce the publication bias by including non-English studies. However due to the small number of the included studies publication bias was still an important limitation in this study. Furthermore, all but one of the studies included in the meta-analysis reported RDW as a significant predictor of mortality. We also did not included one study [7] in which no similar association was found, since it had not reported the sensitivity and specificity.

Studies included in this meta-analysis were quite heterogeneous, which is quite common in the diagnostic test accuracy meta-analyses [26]. Heterogeneity in diagnostic meta-analyses can partly be explained by the variability of cut-off values between studies. However, in our study, the threshold effect analysis showed that it does not contribute to the heterogeneity. Nonetheless, in addition to the small number of studies, there were some differences in the included studies such as patient characteristics, disease severity, etiologies, follow-up periods, study designs and other factors invisible to the authors that might be responsible for the heterogeneity. There is possible effect of etiology, iron deficiency anemia, thalassemia, hemoglobinopathy and other rare factors on RDW. In iron deficiency, RDW is very high and can be a confounding factor, and in these studies they did not evaluate and exclude patients with anemia. Slightly elevated RDW can be seen in hemoglobinopathy and thalassemia, which are not common. Subgroup analysis is commonly used to specifically point out the sources of heterogeneity but could not be performed in this study due to the small number of the included studies. It is also important to note that the bivariate model used in this metaanalysis is considered a random effects model and these models implicitly incorporate some of the heterogeneity observed between studies [29, 35].

Finally, we could not report an ideal cut-off value for RDW to predict mortality since it was beyond the scope of a meta-analysis and requires individual patients data of the included studies. Unlike ordinary ROC curves, it is not possible to identify points on the sROC curve that relate to a particular cut-off with a particular combination of sensitivity and specificity. Additionally, since sensitivity and specificity changes with different cut-off values and the cut-off values of the included studies were different, the reported pooled estimates of sensitivity and specificity in this meta-analysis are not clinically useful and have been reported only to get a sense of sensitivity and specificity relative to each other [26].

\section{Conclusion}

RDW is an easy to use and inexpensive marker that has a moderate prognostic value to predict mortality in AP patients. Clinicians should be more alert when a patient with AP has an increased RDW. More importantly, since RDW is not a strong prognostic marker, we recommend studying its combination with other prognostic markers in an effort to increase their prognostic performance. Furthermore, for a more robust conclusion and to define a precise cut-off value, we recommend a review of individual patient data.

\section{Conflict of Interests}

The authors declare that they have no competing interests.

\section{References}

1. Şenol K, Saylam B, Kocaay F, Tez M. Red cell distribution width as a predictor of mortality in acute pancreatitis. Am J Emerg Med. 2013;31(4):687-9.

2. Kolber W, Sporek M, Dumnicka P, Kuśnierz-Cabala B, Kuzniewski $M$, Gurda-Duda A, et al. [Acute pancreatitis and red cell distribution width (RDW)I at early phase of disease]. Przegl Lek. 2013;70(11):9169.

3. Floreánová K, Dítě $P$, Kianička $B$, Dovrtělová $L$, Přecechtělová $M$. Acute pancreatitis - Validation of Revised Atlanta Classification on 159 patients and prognostic factors. Vnitr Lek. 2014;60(7-8):567-74. 
4. Roberts S, Akbari A, Thorne K, Atkinson M, Evans P. The incidence of acute pancreatitis: impact of social deprivation, alcohol consumption, seasonal and demographic factors. Aliment Pharmacol Ther. 2013;38(5):539-48.

5. Stevenson K, Carter CR. Acute pancreatitis. Surgery - Oxford International Edition.31(6):295-303.

6. Yao J, Lv G. Association between red cell distribution width and acute pancreatitis: A cross-sectional study. BMJ Open. 2014;4(8).

7. Gülen B, Sonmez E, Yaylaci S, Serinken M, Eken C, Dur A, et al. Effect of harmless acute pancreatitis score, red cell distribution width and neutrophil/lymphocyte ratio on the mortality of patients with nontraumatic acute pancreatitis at the emergency department. World Journal of Emergency Medicine. 2015;6(1):29-33.

8. Wang D, Yang J, Zhang J, Zhang S, Wang B, Wang R, et al. Red cell distribution width predicts deaths in patients with acute pancreatitis. J Res Med Sci. 2015;20(5):424-8.

9. Balthazar E. CT diagnosis and staging of acute pancreatitis. Radiol Clin North Am. 1989;27(1):19-37.

10. Pitchumoni CS, Patel NM, Shah P. Factors influencing mortality in acute pancreatitis: can we alter them? J Clin Gastroenterol. 2005;39(9):798-814.

11. Carroll JK, Herrick B, Gipson T, Lee SP. Acute pancreatitis: diagnosis, prognosis, and treatment. Am Fam Physician. 2007;75(10):1513-20.

12. Yang J, Zheng S, Liu HQ, Tang YM. Clinical value of red blood cell distribution width in predicting in-hospital death of patients with severe acute pancreatitis. World Chinese Journal of Digestology. 2016;24(2):305-10.

13. England J, Down M. Red-cell-volume distribution curves and the measurement of anisocytosis. Lancet. 1974;303(7860):701-3.

14. Hu Z, Sun $Y$, Wang $Q$, Han Z, Huang $Y$, Liu X, et al. Red blood cell distribution width is a potential prognostic index for liver disease. Clin Chem Lab Med. 2013;51(7):1403-8.

15. Agarwal S. Red cell distribution width, inflammatory markers and cardiorespiratory fitness: results from the National Health and Nutrition Examination Survey. Indian Heart J. 2012;64(4):380-7.

16. Ani C, Ovbiagele B. Elevated red blood cell distribution width predicts mortality in persons with known stroke. J Neurol Sci. 2009;277(1):103-8.

17. Baynes RD, Bothwell TH, Bezwoda WR, Gear AJ, Atkinson P. Hematologic and iron-related measurements in rheumatoid arthritis. Am J Clin Pathol. 1987;87(2):196-200.

18. Braun E, Domany E, Kenig Y, Mazor Y, Makhoul BF, Azzam ZS. Elevated red cell distribution width predicts poor outcome in young patients with community acquired pneumonia. Crit Care. 2011;15(4):1.

19. Dabbah S, Hammerman H, Markiewicz W, Aronson D. Relation between red cell distribution width and clinical outcomes after acute myocardial infarction. The American journal of cardiology. 2010;105(3):312-7.

20. Hampole CV, Mehrotra AK, Thenappan T, Gomberg-Maitland M, Shah SJ. Usefulness of red cell distribution width as a prognostic marker in pulmonary hypertension. Am J Cardiol. 2009;104(6):868-72.

21. Hong N, Oh J, Kang S-M, Kim S-Y, Won H, Youn JC, et al. Red blood cell distribution width predicts early mortality in patients with acute dyspnea. Clin Chim Acta. 2012;413(11):992-7.

22. Makhoul BF, Khourieh A, Kaplan M, Bahouth F, Aronson D, Azzam ZS. Relation between changes in red cell distribution width and clinical outcomes in acute decompensated heart failure. Int J Cardiol. 2013;167(4):1412-6.

23. Seretis C, Seretis F, Lagoudianakis E, Gemenetzis G, Salemis NS. Is red cell distribution width a novel biomarker of breast cancer activity? Data from a pilot study. J Clin Med Res. 2013;5(2):121-6.

24. Moher D, Liberati A, Tetzlaff J, Altman DG. Preferred reporting items for systematic reviews and meta-analyses: the PRISMA statement. Ann Intern Med. 2009;151(4):264-9.

25. http://www.cebm.net/wp-content/uploads/2014/04/cebmprognosis-worksheet.pdf. Accessed 20 Jan 2016.

26. Bossuyt P, Davenport C, Deeks J, Hyde C, Leeflang M, Scholten R. Chapter 11: Interpreting results and drawing conclusions. Cochrane handbook for systematic reviews of diagnostic test accuracy version 09 The Cochrane Collaboration. 2013.
27. Reitsma JB, Glas AS, Rutjes AW, Scholten RJ, Bossuyt PM, Zwinderman $\mathrm{AH}$. Bivariate analysis of sensitivity and specificity produces informative summary measures in diagnostic reviews. J Clin Epidemiol. 2005;58(10):982-90.

28. Doebler P, Holling $H$. Meta-analysis of diagnostic accuracy with mada. Reterieved from:

https://cran.rproject.org/web/packages/mada/vignettes/mada.pdf 2015.

29. Macaskill P, Gatsonis C, Deeks J, Harbord R, Takwoingi Y. Cochrane handbook for systematic reviews of diagnostic test accuracy. Version 090 London: The Cochrane Collaboration. 2010.

30. Zamora J, Abraira V, Muriel A, Khan K, Coomarasamy A. Meta-DiSc: a software for meta-analysis of test accuracy data. BMC Med Res Methodol. 2006;6(1):31.

31. Guo Z-h, Hao J-y. Review of acute pancreatitis scoring systems. Journal of Clinical Hepatology. 2011;11:009.

32. Wu BU, Johannes RS, Sun X, Conwell DL, Banks PA. Early changes in blood urea nitrogen predict mortality in acute pancreatitis. Gastroenterology. 2009;137(1):129-35.

33. Lappé JM, Horne BD, Shah SH, May HT, Muhlestein JB, Lappé DL, et al. Red cell distribution width, C-reactive protein, the complete blood count, and mortality in patients with coronary disease and a normal comparison population. Clin Chim Acta. 2011;412(23):2094-9.

34. Fukuta H, Ohte N, Mukai S, Saeki T, Asada K, Wakami K, et al. Elevated plasma levels of B-type natriuretic peptide but not Creactive protein are associated with higher red cell distribution width in patients with coronary artery disease. Int Heart J. 2009;50(3):30112.

35. Sousa MRd, Ribeiro ALP. Systematic review and meta-analysis of diagnostic and prognostic studies: a tutorial. Arq Bras Cardiol. 2009;92(3):241-51. 